\title{
Automated generation of rotation-robust corner detectors
}

\author{
Kisung Seo $^{\text {a) }}$ and Youngkyun Kim \\ Dept. of Electronic Engineering, Seokyeong University \\ Jungneung-Dong 16-1, Sungbuk-Gu, Seoul 136-704, KOREA \\ a)ksseo@skuniv.ac.kr
}

\begin{abstract}
This paper introduces evolutionary generation method of robust corner detectors for rotated images. Previous Harris, SUSAN and FAST corner detectors are highly efficient for well-defined corners, but frequently mis-detect as corners the corner-like edges which are often generated in rotated images. In this paper, we have focused on this challenging problem and proposed using Genetic Programming to do automated generation of corner detectors that work robustly on rotated images. Especially, a well-devised terminal set is proposed based on intensity-related information, several mask sizes, and amount of contiguity of neighboring pixels of similar intensity. This method is then compared to three existing corner detectors on test images and shows superior results.
\end{abstract}

Keywords: corner detector, automated generation, genetic programming, rotation robust

Classification: Science and engineering for electronics

\section{References}

[1] H. P. Moravec, "Visual mapping by a robot rover," Proc. 6th International Joint Conf. Artificial Intelligence, IJACI, Tokyo, pp. 598-600, 1979.

[2] C. Harris and M. Stephens, "A Combined Corner and Edge Detector," Proc. 4th Alvey Vision Conf., UK, pp. 147-151, 1998.

[3] S. M. Smith and J. B. Brady, "SUSAN-A New Approach to Low Level Image Processing," Int. J. Computer Vision, vol. 23, no. 1, pp. 45-78, 1997.

[4] E. Rosten, R. Porter, and T. Drummond, "Faster and better: a machine learning approach to corner detection," Pattern Analysis and Machine Intelligence, vol. 32, no. 1, pp. 105-119, 2008.

[5] J. R. Koza, Genetic Programming: On the Programming of Computers by Natural Selection, MIT Press, Cambridge, MA, 1992.

[6] K. Seo and Y. K. Kim, "Scale- and Rotation-Robust Genetic Programming-Based Corner Detectors," EvoApplication 2010, Part 1, LNCS 6024, pp. 392-401, 2010.

[7] S. Silva, GPLAB: A Genetic Programming Toolbox for MATLAB, Ver. 3, [Online] http://gplab.sourceforge.net/index.html

[8] F. Mokhtarian and F. Mohanna, "Performance evaluation of corner detectors using consistency and accuracy measures," Computer Vision and 


\section{Introduction}

Corner detection is used for image matching, recognition, tracking, and SLAM (simultaneous localization and mapping). Many algorithms for corner detection have been introduced in computer vision research. Moravec [1] developed an interest point detector based on the auto-correlation function. Harris and Stephens [2] modified the Moravec method and proposed the famous Harris corner detector by estimating the autocorrelation from the firstorder derivatives. Smith and Brady [3] proposed a corner detection algorithm known as the SUSAN corner detector based on brightness comparison and a corner mask in a circular window. Rosten and Drummond [4] introduced the FAST (Features from Accelerated Segment Test) feature detector. This recent approach shows good performance in repeatability and efficiency in real world application.

However, those competitive algorithms may detect false corners when the boundaries are step-like or there is a small change in slope which just represents an edge. Edge pixels have similar characteristic with corner pixels, so this phenomenon can occur after rotation of images.

We propose Genetic Programming-based automated generation of corner detectors which is robust to rotational transformation. Efficient terminal sets are introduced to capture the key properties of corners, combining intensityrelated information, several mask sizes, and contiguity of neighboring pixels allows a well-devised terminal set to be proposed. The proposed method is compared to existing corner detectors and rotated test images.

\section{Problem and proposed method}

\subsection{Problem statements}

Most existing algorithms perform well for well-defined corners in images. However, some corners are ill-defined because of noise and some non-corners are often misclassified as corners for example, inclined edge. Especially corner-like edges are often generated when an image is rotated. It is very difficult to detect correctly these edges which have characteristics similar to corners. In this paper, we have focused on the challenging problem above and proposed a Genetic Programming-based automated generation of corner detectors that is robust to rotation of images.

\subsection{Genetic programming}

Genetic programming [5] is an extension of the genetic algorithm, using evolution to optimize actual computer programs or algorithms to solve some task, such as automated synthesis. Genetic programming can manipulate variable-sized entities and can be used to "grow" trees that specify increasingly complex corner detectors, as described below. In our experience, it 
is very important in corner detection to define function and terminal sets carefully. The terminal set seems to be especially critical, because it is more closely related to physical characteristics of corner points than is the function set, which usually represents general-purpose operators.

\subsection{GP function and terminal set}

The function set for the proposed GP-based detector involves 5 arithmetic operator and 3 conditional operators as follows.

$$
\mathrm{F}=\{+,-, \times, \div, \text { abs, if, } \min , \max \}
$$

The set of primitive functions has to be sufficient to allow solution of the problem at hand, but there are typically many possible choices of sets of operators that meet this condition. For corner detection, the choice of function set seems to be less critical than the choice of terminal set.

Below, an efficient terminal set is presented to capture the key properties of corners, the proposed GP method, which is refined from [6].

For each pixel in a rectangular mask, the intensity of the pixel relative to the center pixel is classified into one of darker, similar, or brighter states. Second, different sizes of rectangular masks are used $3 \times 3,5 \times 5$, and $7 \times 7$. Third, the number of pixels of similar intensity in contiguous region is added. The terminals are defined combining these three intensity, size measures of masks, and contiguity as shown in Figure 1.

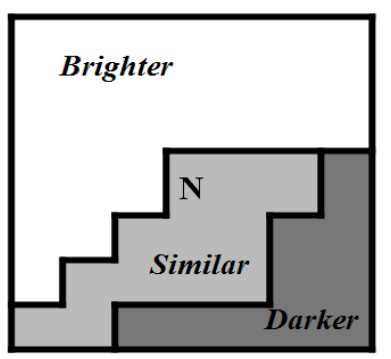

(a)

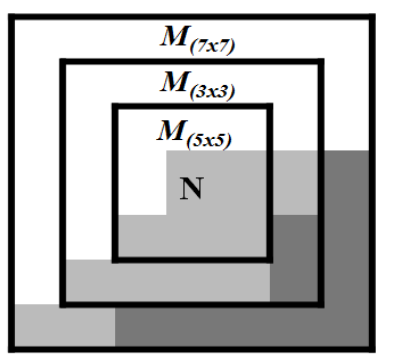

(b)

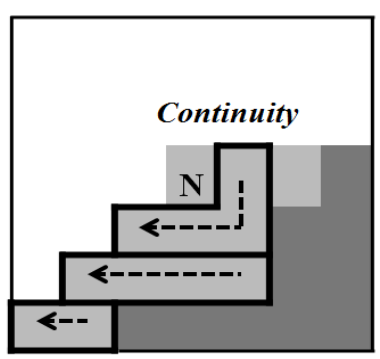

(c)

Fig. 1. Terminals based on (a) intensity, (b) sizes of rectangular masks, and (c) continuity.

\subsection{Fitness function}

The goal of corner detection in our paper is to achieve robust performance under rotation. First, both MR (Miss Rate not finding a corner) and FPR (False Positive Rate calling a feature a corner that is not) are used as basic indexes below. RI (Rotated Index) are defined based on MR and FPR. The fitness function is based on a combination of the MR and the FPR under rotational transformations. The coefficient $\alpha$ and $\beta$ are weighing value for $R I_{M R}$ and $R I_{F P R}$.

The fitness of a genetic program is obtained as follows. Apply the program as a moving $n \times n$ window template ( $n$ is the size of the input field) to 
each of the training images and obtain the output value of the program at each possible window position. Match the detected corners with the known locations of each of the desired true corners. Calculate the fitness function of the evolved GP tree as follows in equation (1):

$$
\begin{aligned}
& M R=(\text { miss } / \text { total_corners }) \times 100 \\
& F P R=(\text { false } / \text { total_non }- \text { corners }) \times 100 \\
& \text { Fitness Function }=\left(\alpha \times R I_{M R}+\beta \times R I_{F P R}\right)
\end{aligned}
$$

\section{Experiments and Analysis}

\subsection{Experimental setup}

We use artificial images in the first experiments (Figure 2 (a) in section 3.2). The original image includes many corner types, including L-Junctions, Y-Junctions, T-Junctions, Arrow-Junctions and X-Junctions. It has been widely used to evaluate corner detectors. Many corner-like edges are generated when the image is rotated. We have tested various images rotated by 30 , 45 degrees. The GP programs were run on a Pentium Core2Duo $2.66 \mathrm{GHz}$ with 4 GB RAM using GPLAB ver.3. [7] The GP parameters used for the corner detector design were as follows:

Number of generations: 100 ,

Max depth: 7, 10, 12, 17

Crossover: 0.8
Population sizes: 100, 200, 300

Selection: Tournament $($ size $=7$ )

Mutation: 0.2

\subsection{Experimental comparison with existing methods}

Below, the proposed GP-based corner detector is compared to several typical corner detectors, namely Harris, SUSAN, and FAST, on all five test images. The consistency of corner numbers (CCN) and accuracy (ACU) [8] were used for measuring the performance of corner detectors for the rotated images.

$$
C C N=100 \times 1.1^{-\left|N_{t}-N_{o}\right|}
$$

where $N_{o}$ is the number of corners in the original image and $N_{t}$ ) is the number of corners in the transformed image $\left(N_{t}\right)$. CCN should be close to $100 \%$ for accurate corner detectors [8].

$$
A C U=100 \times\left(\frac{N_{a}}{N_{o}}+\frac{N_{a}}{N_{g}}\right) / 2
$$

where $N_{o}$ is the total number of corners detected by a specific corner detector, $N_{g}$ is the total number of corners in the ground truth data, and $N_{a}$ is the total number of matching corners when comparing the first set of corners. The value of ACU for accurate corner detectors should be close to $100 \%[8]$.

Table I (top) shows the CCN values of rotated $\left(30^{\circ}, 45^{\circ}\right)$ images for four methods: Harris, SUSAN, FAST, and the proposed GP-based method. The CCN of Harris dropped to zero in $30^{\circ}$ rotated images, averaging 54.7 overall. In SUSAN, all rotated CCN values were close to zero and showed the worst 
result. The CCNs of all transformed images in FAST showed similar values which were around 40. The average of the CCNs for FAST was 61.6, which was better than was obtained by Harris or SUSAN. These results means that the three previously existing corner detectors failed to satisfy consistency for rotated images. However, the proposed GP-based detector maintained much better CCN values for all transformed images; the average was 83.77. Thus the new method proved to be superior in consistency to the other approaches for this test dataset.

Table I. Comparison results for the CCN consistency criterion and the ACU criteria

\begin{tabular}{c|c|c|c|c}
\hline CCN (\%) & Original & $30^{\circ}$ & $45^{\circ}$ & Average \\
\hline \hline Harris & 100 & 2.01 & 62.09 & 54.70 \\
\hline SUSAN & 100 & 5.73 & 2.94 & 36.22 \\
\hline FAST & 100 & 42.41 & 42.41 & 61.60 \\
\hline GP & 100 & 51.32 & 100 & 83.77 \\
\hline
\end{tabular}

\begin{tabular}{c|c|c|c|c}
\hline ACU (\%) & Original & $30^{\circ}$ & $45^{\circ}$ & Average \\
\hline \hline Harris & 87.97 & 74.24 & 87.89 & 83.36 \\
\hline SUSAN & 94.50 & 83.43 & 80.38 & 86.10 \\
\hline FAST & 95.46 & 93.96 & 92.72 & 94.05 \\
\hline GP & 98.75 & 94.83 & 94.83 & 96.13 \\
\hline
\end{tabular}

Table I (bottom) shows results for ACU values. Similarly to the CCN results, the ACU of Harris and SUSAN represented lower average values $83.36 \%$ and $86.1 \%$, respectively. The ACU of FAST had better results than Harris and SUSAN in all images, with $94.05 \%$. The ACU of the proposed detector was much better, averaging $96.13 \%$ across all images.

The proposed detector clearly outperformed the others. Therefore it is verified that the proposed detectors' performance was superior to existing detectors on the rotated transformed images tested. Figure 2 shows the corner maps for both artificial images (a) and natural images (b) obtained by the Harris, SUSAN, FAST, GP-based corner detectors. We can see that Harris and SUSAN detectors mis-detect as corners many corner-like edges in rotated images, especially as shown in the first and second columns of Figure 2 (a). The FAST detector reduced sharply the false detection of these corner-like edges, but missed several actual corners in rotated images. The GP-based corner detector shows the best performance in both false positives and false negatives as shown on the far right of Figure 2 (a). Similar results of additional experiments for natural images (blocks and house) are shown in Figure 2 (b). 

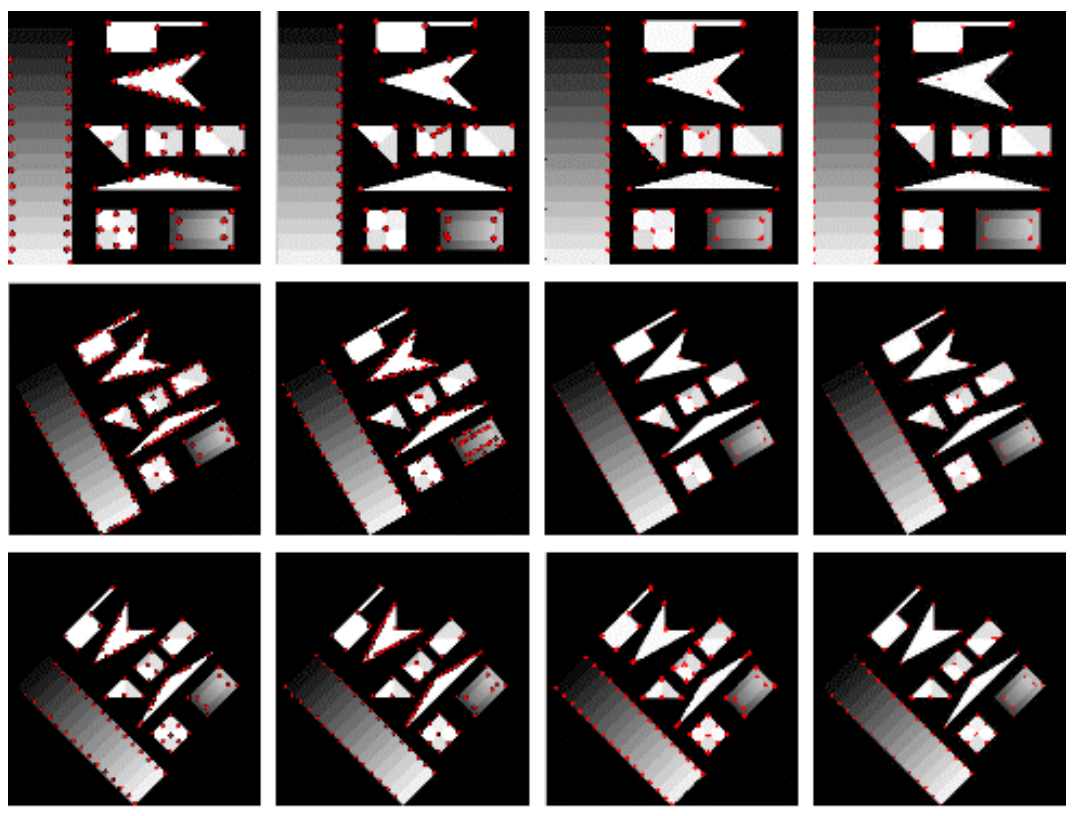

(a)
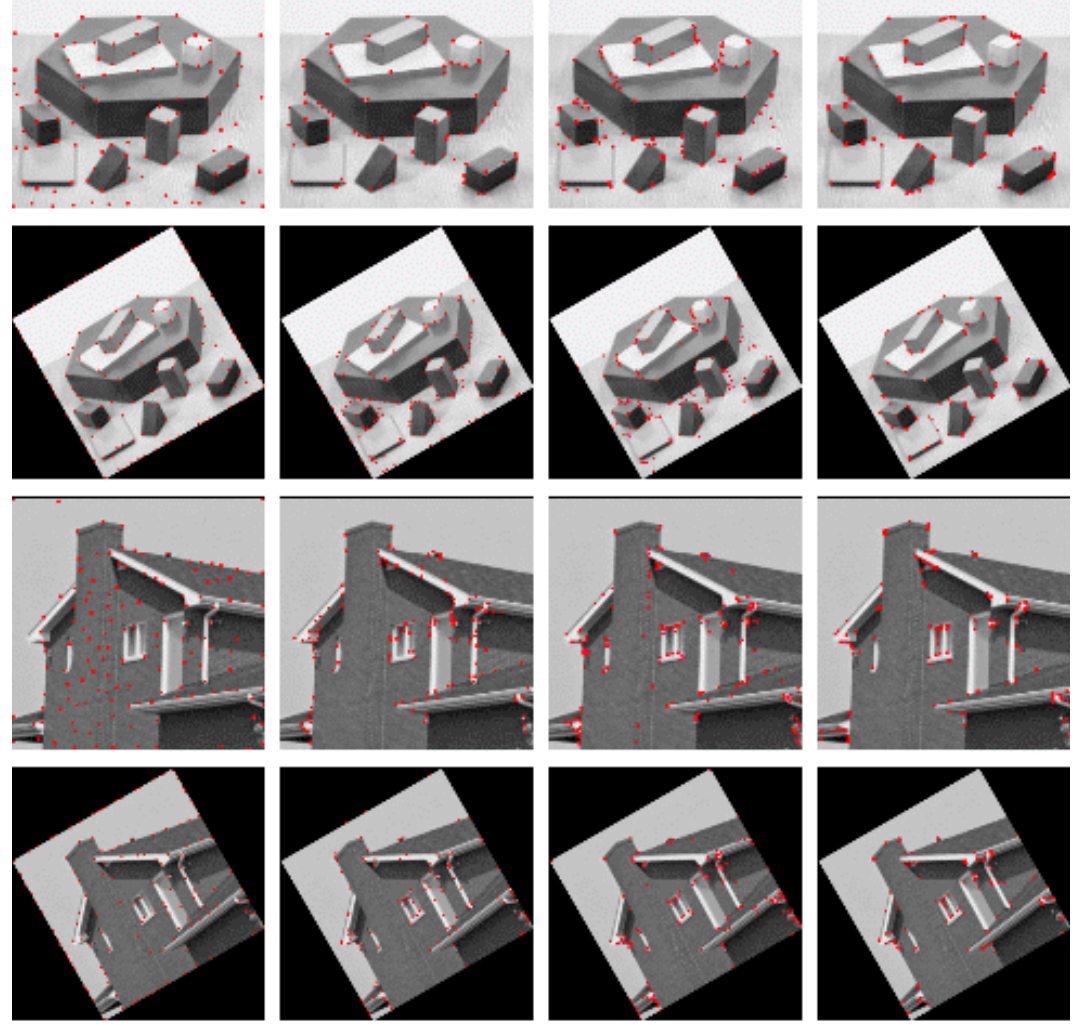

(b)

Fig. 2. (a) Result images of corner maps for artificial images (Original, $30^{\circ}, 45^{\circ}$ ) transformed images from top, and Harris, SUSAN, FAST, GP-based corner (b) Result images of corner maps for natural blocks and house images (Original, $30^{\circ}$ ) transformed images from top, and Harris, SUSAN, FAST, GP-based corner detector form left. 


\section{Conclusions}

GP- (Genetic Programming-) based robust corner detectors for rotated images are presented. Our approach posed corner detection as an optimization problem, and developed an evolution methodology which enables automatic generation of corner detectors. GP terminals and functions were carefully designed to discriminate corners correctly from noise and corner-like edges. Combining intensity-related information, several mask sizes, and continuity of neighboring pixels allowed generation of a high-performance terminal set. The proposed GP-based corner detector is compared to three typical existing corner detectors, Harris, SUSAN and FAST, on a test images and rotated versions. Performance measures were selected to evaluate accuracy and robustness for rotated images. Experimental results showed that the proposed approach generated a superior corner detector to those of existing methods for both artificial and natural images. Further study will aim at refinement of the GP representation, improvement of the GP evolution, and application to more complex natural images.

\section{Acknowledgments}

This Research was supported by Seokyeong University in 2009. 